\section{The Canadian Standards Association Technical Committee Z252.11 for Lab- developed Tests provides information}

In response to the 2019 CMAJ commentary by Holloway and colleagues, ${ }^{1}$ we wish to clarify:

- the role of the Canadian Standards Association (CSA) in standards development;

- the link between voluntary standards and laboratory accreditation requirements; and

- the financial and in-kind support received for the development of the CSA standard referenced in the commentary.

The CSA (operating as "CSA Group") is a private, not-for-profit company that publishes voluntary standards and related documents through a consensusbased standards development process approved by the Standards Council of Canada. This process brings together volunteers who represent varied viewpoints and interests to achieve consensus.

The first edition of CSA Z316.8:18 requirements for the design, development and validation of laboratory-developed tests used for the screening, diagnosis and management of clinical conditions assists in achieving harmonization of the management of laboratory-developed tests. The standard provides guidance to laboratories and assists accreditation bodies in the establishment of a standardized validation process.

The standard provides a distinction between testing done in a research setting versus in a clinical setting. A laboratory-developed test is intended to assist in clinical diagnosis or to be used in making decisions concerning clinical management. This test is a type of in vitro diagnostic test that is designed, created, validated and used within a single laboratory. Laboratory-developed tests can be shared by paternal laboratory networks (including technology transfer from provincial/territorial, federal and private laboratories), which are frequently involved in the maintenance of test-performance features. However, the ultimate test performance is the responsibility of a single laboratory. Tests clearly labelled and marketed for research use only by the manufacturer may be used in a clinical setting for diagnosis or managing treatment only if they are technically and clinically validated within the laboratory carrying out the testing. Once validated for clinical use, a test labelled as research use only is considered a laboratory-devloped test and can be used in the clinical laboratory. The standard does not apply to tests that are developed by a laboratory for research purposes until such time that the test becomes applied to clinical testing and has undergone suitable validation according to the requirements in the CSA standard.

Laboratories validate laboratorydeveloped tests by establishing acceptable test-performance requirements through analytical and clinical testing. Laboratories then reverify performance as part of implementation, including precision, accuracy, bias, limit of detection, linearity, selectivity, analytical specificity, stability, dilution effects, carryover, reference/therapeutic range and clinical validation. The measuring and reference intervals should be determined. A validation summary should be written by the laboratory to summarize experiments and determine acceptance criteria, equivalent to the package insert for a commercial approved test.

A voluntary standard, such as those produced by CSA Group, can be referenced by an accreditation body, which may choose to mandate the standard in its entirety or certain clauses to assess laboratory compliance with the standard. For instance, the Institute for Quality Management in Healthcare accreditation body incorporated the CSA Z316.8:18 standard into its accreditation requirements in December 2019, which took effect for all accreditation assessments as of Jan. 1, 2020. The institute program is mandatory in 3 Canadian provinces: Ontario, New Brunswick and Newfoundland and Labrador. The CSA
Z316.8:18 standard is also being implemented by the College of Physicians and Surgeons of Alberta via the Western Canada Diagnostic Accreditation Alliance.

Members of CSA technical committees and subcommittees provide technical expertise in the content development of standards; they do not act on behalf of the association or organization where they are employed. Financial support to develop the standard was provided, in part, from the governments of the provinces and territories, as administered by the Canadian Agency for Drugs and Technologies in Health, Canadian Society for Medical Laboratory Science, Alere ULC, Siemens and Roche, and developed in accordance with CSA Group's accredited process. The standard was developed by voluntary members of the technical subcommittee for laboratory-developed tests and approved by the technical committee. Members represent their respective stakeholder groups and are chosen based on their experience relevant to these groups.

The technical subcommittee accounted for the needs of regulatory authorities, consumers, contractors, providers of related services and other users when developing the technical content of the standard. The standard was developed in such a way that it could be adopted by regulators and to lay the groundwork for potential future development. When the requirements in the standard were developed, the technical subcommittee determined the best technical resolution to provide the minimum guidelines that any affected stakeholder could implement to ensure best safety practices. At the time of development, this standard was intended to provide a basis for the implementation of a consistent laboratory-developed testing development process across Canada. The technical subcommittee is currently reviewing the potential for additional standard development in the genetic topic area.

Problematic laboratory-developed tests in the onset of the coronavirus disease 2019 pandemic are the result of fast response for the emergent medical need. The referenced example is a Centers for 
Disease Control and Prevention qPCRbased test design for severe acute respiratory syndrome coronavirus 2 acute infection (www.sciencemag.org/news/2020/02/ united-states-badly-bungled-coronavirus -testing-things-may-soon-improve). This was corrected within a few days thanks to the decentralized and parallel modality laboratory-developed tests that were produced and transparently compared in real time among laboratory professionals. This offers the best mode to reach international consensus in performance in the current pandemic situation. Health Canada regulates in vitro diagnostic tests; a manufacturer has to apply for approval before being allowed to market and sell the test kits or reagents in Canada.
Health Canada's jurisdiction does not extend to tests developed within Canadian clinical laboratories that are not intended for sale or distribution to other laboratories. Regulation at this level rests with each provincial health ministry, and each province has its own system for regulation of clinical laboratories. The necessary oversight of laboratory-developed tests by accreditation of laboratories varies according to provincial accreditation programs' requirements.

At the time of development, this standard was intended to provide a basis for the implementation of a consistent development process for laboratory-developed testing across Canada. It provides guidance to both the laboratories developing tests for use in their own laboratories, as well as for accreditation bodies.

\section{Edward Dunn PhD}

Director, Product and service

development, Dynacare, Brampton, Ont.

\section{Robert Rennie PhD}

Provincial microbiology consultant, RP Rennie Consultations Ltd.; Professor emeritus, Department of Laboratory Medicine and Pathology, Faculty of Medicine and Dentistry, University of Alberta, Edmonton, Alta.

- Cite as: CMAJ 2020 October 5;192: E1166-7. doi: 10.1503/cmaj.76509

\section{Reference}

1. Holloway K, Miller FA, Rousseau F, et al. Health Canada needs to act on laboratory-developed diagnostics. CMAJ 2019;191:E1067-9.

Competing interests: None declared. 